\title{
EL BULLYING, CONTEXTUALIZADO EN DOS UNIDADES EDUCATIVAS BÁSICAS DEL CANTÓN DE SANTA ELENA - ECUADOR
}

\section{THE BULLYING, CONTEXTUALIZED IN TWO BASIC EDUCATIONAL UNITS OF THE CANTON OF SANTA ELENA - ECUADOR}

\author{
Carlota Ordoñez Villao ${ }^{1}$ \\ Javier Mogrovejo ${ }^{1}$ \\ Paola Peralta ${ }^{1}$ \\ Narcisa González ${ }^{1}$ \\ ${ }^{1}$ Facultad de Educación e Idiomas, Universidad Estatal Península de Santa Elena, Ecuador. \\ delroz2370@yahoo.com , javiermogrovejo@yahoo.es
}

\section{RESUMEN}

El objetivo de este reporte científico consiste en argumentar de qué manera el bullying incide en el aprendizaje y la conducta de los educandos de dos escuelas de la provincia de Santa Elena, donde se evidencia aptitudes y acciones negativas dentro y fuera del aula de clases. Para efecto se aplicó el test de Bull a una muestra de 90 estudiantes de quinto grados de Educación Básica Elemental, se recopiló antecedentes de conductas y actitudes que luego del análisis este fenómeno interviene en los diferentes espacios educativos presentándose en formas de acosamientos físicos, verbal y psicológicos, se evidencia como un factor de riesgo para la integridad y el aprendizaje de los niños y niñas de ambos Centros Educativos. Finalmente los resultados obtenidos describen que el bullying en una realidad que se incorpora con más frecuencia, y que por razones de desconocimiento; falta de modelos de prevención y de escasos recursos limitan la intervención oportuna para mejorar de forma adecuada la convivencia e inclusión escolar.

Palabras clave: Bullying, conductas, prevención, aprendizaje.

\section{ABSTRACT}

The objective of this scientific report is to explain how bullying affects learning and the students' behavior of two schools located in Santa Elena province, where it is evident aptitudes and negative actions inside and outside of the classroom. For this, it was applied the Bull test to 90 students of fifth grade of Basic Elemental Education, there was collected a background of behavior and attitudes that after the analysis this phenomenon intervenes in the different educational environments presenting in forms of physical and psychological harassment, evidence as a risk factor for the integrity and learning of the children in both Educational Institutions. Finally, the results obtained describe that Bullying is a reality that is incorporated more frequently, and because of the lack of knowledge and prevention models as well as scarce resources limit the appropriate intervention to improve adequately coexistence and school inclusion.

Keywords: bullying behavior, prevention, learning

Recibido: 31 de enero de 2017

Aceptado: 13 de julio de 2017

Publicado: 31 de julio de 2017 


\section{Introducción}

La existencia de espacios seguros donde se integran nuestros hijos y que estos se encuentren tranquilos, aceptados y acogidos son requerimientos que todos los padres deseamos, por otro lado los docentes en sus expectativas en tener un grupo de niños tranquilos en armonía y equilibrados, sin problemas de conducta y de aprendizaje se convierte en la utopía del docente cuando reciben a los estudiantes.

En las instituciones educativas actualmente ingresan niños de diferentes extractos sociales, con diversos estilos de aprendizaje y de comportamientos. De acuerdo a las indagaciones realizadas en las dos instituciones asisten niños con familias disfuncionales, con problemas de violencia familiar y un porcentaje minoritario son familias funcionales. Los planteles a pesar de tener un plan académico diseñado para el proceso lectivo y debido al aumento de estudiantes por aulas no han podido minimizar la problemática $y$ las situaciones de Bullying, acoso y/o violencia se han evidenciado en el comportamiento y aptitudes de un grupo de estudiantes.

Dan Olwes en $1973^{1}$, fue el primero en abordar en sus investigaciones el termino bullying, pero en la década de los sesenta inicio con mayor profundidad el estudio de este fenómeno, en Ecuador a través de la Leyes Generales del Ministerio de Educación en sus acuerdos 3412 pretende orientar de manera pertinentes este problema, debido a que $60 \%$ de los niños han sufrido acosos y de acuerdo a datos es el segundo país con mayor porcentaje.

Organismos como en Ministerio de Inclusión Económica y Social (MIES) en su política de estado junto al Ministerio de Educación vela por la protección infantil y ante esta situación señala:

"En los últimos cinco años, el Bullying o acoso escolar se ha convertido, dentro del imaginario social, en uno de los riesgos más graves que tienen los niños y niñas. A diferencia de la violencia en el aula, implica un acoso continuo dentro y/o fuera de ésta que se agudiza cuando se utilizan las tecnologías de la información. El acoso se hace público, rebasa el aula de clases $y$ es un sometimiento del más fuerte al más débil, que mina la capacidad de respuesta. Ante esta nueva expresión de la violencia, el país apenas comienza a reaccionar".

Este fenómeno denominado bullying debe ser atendido, buscar las estrategias necesarias para minimizar el riesgo para mejorar el ambiente social e integrado; donde con la colaboración de docentes; padres; estudiantes y comunidad se armonice la convivencia y se de inclusión educativa.

En el 2014 la Universidad UPSE a través de la Carrera de Educación Básica realiza el proyecto de Vinculación con la Comunidad previo a las observaciones In situ, destacan que la problemática del bullying es una acción sistemática donde los estudiantes con mayor poder minimizan el autoestima desvalorizando de manera gradual desprotegiendo a ese ser agredido confundido el cual no tiene la capacidad para defenderse.

En los Centro de Educación Básica Veinticuatro de Julio y Teodoro Wolf del cantón Santa Elena, se detecta situaciones más comunes como agresiones físicas, seguidas de los insultos, amenazas, conductas negativas que son repetitivas entre niños de 8 a 12 años. Por lo que la investigación se dirige a determinar el grado de incidencia de bullying entre los variables convivencia escolar y aprendizaje, analizar los efectos psico-socio-educativos que genera el bullying mediante técnicas como: grupo focal, encuestas, aplicación del test de Bull para luego proceder a diseñar un proyecto donde se construya una estrategia de prevención e intervención en el manejo de esta problemática social y mejorar la convivencia de inclusión escolar.

Al observar en los diferentes espacios de las dos instituciones se detecta escasos espacios seguros, se confrontan situaciones de relaciones interpersonales inadecuadas en acciones y aptitudes negativas de índole física, verbal, emocional. Convirtiéndose los espacios principalmente las áreas abiertas en un nivel alto de acoso e intimidación.

Las horas de descanso se convierten en el desafío de sobrevivir frente a la marginación, intimidación y segregación, avanzan con mofa, agresiones y en situaciones más graves violencia, insultos y robos sean esta por niños de su propia edad o por jóvenes.

El bullying es un tema conocido pero que aún falta aborda de manera pertinente en las escuelas, con las orientaciones propias, a su vez la perspectiva de docentes, padres, familia y comunidad, le dan escasa importancia a las situaciones emocionales que puede sufrir sus hijos, este desafío tal vez se impone 
principalmente porque no hay políticas claras de intervención en lo que se refiere a la salud mental y una cultura psicológica de la prevención en relación al fenómeno bullying.

El bullying es una situación que incide en su accionar dentro del círculo familiar, porque muchas veces toma forma de situaciones de maltrato, intimidación, violencia, incomodidad $u$ otra que afecta y que luego se proyecta en el aula de clase o en otros ambientes. Muchas expresiones se ven reflejadas en el comportamiento con aptitudes demostradas de burla, uso de apodos, apelativos, malas palabras, provocaciones hasta las acciones más denigrantes como cyberbullying, hostigamiento, exclusión de grupos y hasta la pérdida de la propia vida.

De acuerdo a la revista CEPAL (2014) el género y la edad inciden en la expresión y magnitud que alcanza el acoso entre compañeros. Así, los estudiantes varones se ven envueltos mayormente en situaciones de maltrato físico (golpes), mientras que las mujeres ocupan preferentemente el maltrato social o psicológico (Skrzypiec, 2008).

La UNESCO en un estudio realizado durante los años 2009 al 2011 refiere: "las familia de latino américa en algún momento han sido víctimas del fenómeno denominado Bullying o conocido como acoso, hostigamiento, el porcentaje es de 51,1\%". Así con este porcentaje en Ecuador la violencia entre los estudiantes es un fenómeno considerado como normal en el diario vivir entre ellos.

La CEPAL (2012) demuestra que en el Ecuador respecto a la violencia física entre estudiantes alcanza un $21.9 \%$ al realizar una comparación entre la convivencia escolar y su desempeño académico este fenómeno se refleja en la inclusión de las diversas actividades que se dan en los diversos contextos educativos.

La Organización Mundial de la Salud (OMS) define a la violencia como "el uso intencional de la fuerza física o el poder contra uno mismo, hacia otra persona, grupos o comunidades y que tiene como consecuencias probables lesiones físicas, daños psicológicos, alteraciones del desarrollo, abandono e incluso la muerte"(Semfyc, 2007).

El bullying puede tener consecuencias a largo plazo tanto académico, físico, emocional en los bullies, sus agredidos y testigos. La incidencia del bullying en las escuelas tiene un impacto negativo en la oportunidad de los estudiantes de aprender en un ambiente seguro y donde ellos sean tratados con respeto. (Turner, 2004). Herreras, B. (2008) enfatiza sobre la difusión del mismo y enuncia: "es apenas ahora, en las últimas décadas cuando esta violencia empieza a ocupar los espacios de la comunicación y el debate público, cuando es una realidad presente desde hace décadas".

Uno de los aspectos irracionales que podemos considerar es que el bullying solo puede darse entre adultos, y por los resultados obtenidos se puede constatar que es un fenómeno social y comportamental que se puede fraguar a cualquier edad, siendo más impactante cuando se lo ejecuta con una persona inocente e indefensa.

Muchos de los integrantes de la comunidad escolar, directivos, profesores y padres, tienen dificultades para definir a la violencia y en la mayor parte de los casos lo asocian con la violencia física especialmente con la televisión. (Cisneros \& Gallegos G., 2010)

Díaz Aguado (2005, p. 18) identifica tres características que contribuyen a la violencia escolar: (i) la justificación o permisividad de la violencia como forma de resolución de conflictos entre iguales; (ii) el tratamiento habitual que se da a la diversidad actuando como si no existiera y (iii) la falta de respuesta del profesorado ante la violencia entre escolares, que deja a las víctimas sin ayuda y suele ser interpretada por los agresores como un apoyo implícito.

En los centros educativos es muy común observar los diferentes tipos de relaciones sociales y específicamente en el trato entre niños, demostrando actitudes y comportamiento que reflejan conductas que en muchos casos con violencia, esto es una realidad de convivencia natural que puede ser registrada en los diferentes espacios de las instituciones educativas, a veces sin ser percibida por el docente y que pueden provocar niveles bajo de aprendizaje logrando excluirse del medio social y educativo.

El niño intimidado, agredido, ridiculizado se aísla en su comportamiento autoguardando sentimientos de temor a todo, ansiedad y estrés por situaciones aun no entendidas del riesgo y la incertidumbre de no ser comprendido, esta problemática se verá reflejada en el proceso de su aprendizaje.

De acuerdo a Sánchez L. (2009, 14), “La incidencia del fenómeno bullying es similar en todos los países y en todos los sistemas educativos, independientemente de su cultura y su sistema educativo". Los diferentes acontecimientos que se han dado en el Ecuador y se reportan por los medios de comunicación nos permiten tomar con mayor 
seriedad el abordaje y prevención de este malestar social, ejecutar de una forma clara y adecuada estrategias que se guíen por reglas, normas y políticas realizables y adecuadas a la realidad cultural de nuestro país donde todos seamos actores proactivos de la lucha contra este problema.

La medicina familiar define al bullying como la conducta de persecución física y/o psicológica que realiza un alumno, o varios contra otro, al que escoge como víctima de repetidos ataques. (Semfyc, 2007). Esta situación de riesgo percibido por la víctima no le permitirá cognitivamente enfocarse en el aprendizaje al sentirse vulnerable su rendimiento escolar estará sumidos en sus temores y centrados en la ansiedad del acoso.

Por tal motivo es de donde en algún momento los docentes y profesionales del campo deberán enfocar su mirada a construir y fortalecer las emociones familiares para una mejor convivencia de niños y jóvenes en ambientes sanos, normales adecuados a su pleno desarrollo y satisfacción física psicología social.

\section{Metodología}

Atendiendo a la realización de la contextualización del Bullying se realizó un estudio de carácter exploratorio y para complementar los datos se aplicó el Test Bull instrumento del GRUPO ALBOR-COHS, adaptado a la realidad del contexto de las instituciones involucradas, escenario, manifestaciones de emociones y sentimientos, acciones que referencien si hay o no bullying. Se aplicó este cuestionario a 123 estudiantes de los ciclo 1 y 2 de Educación Básica Elemental, edades comprendidas entre 6 a 9 años

Tabla 1: Variables de la investigación contextualización del bullying

\begin{tabular}{|c|c|c|c|c|c|}
\hline \multirow{2}{*}{\multicolumn{2}{|c|}{ Género }} & \multicolumn{2}{|c|}{ Rango de Edad } & \multicolumn{2}{|c|}{ Nivel o grado } \\
\hline & & 6 a 8 & 8 a 10 & 2do & 3ero \\
\hline Hombres & 137 & 123 & 11 & 85 & 52 \\
\hline Mujeres & 111 & 109 & 6 & 84 & 27 \\
\hline Total & 248 & 232 & 16 & 169 & 79 \\
\hline
\end{tabular}

\section{Resultados}

A continuación se ilustran los resultados obtenidos en el proceso de investigación sobre el Bullying, emanados de la encuesta aplicada.

Tabla 2: Espacios donde se presentan situaciones de Bullying escolar

\begin{tabular}{|l|c|}
\hline \multicolumn{1}{|c|}{ AMBAS INSTITUCIONES } & TOTAL \\
\hline Aula de clases & 43 \\
\hline Patio & 41 \\
\hline Pasillos & 1 \\
\hline Bar o comedor & 3 \\
\hline En una asignatura en concreto & 1 \\
\hline Baños & 0 \\
\hline Lugares alejados dentro de la misma IE & 0 \\
\hline Por las redes sociales & \\
\hline
\end{tabular}

Tabla 3: Frecuencia de agresiones

\begin{tabular}{|l|c|}
\multicolumn{1}{|c|}{ AMBAS INSTITUCIONES } & TOTAL \\
\hline Todos los días & 128 \\
\hline $1-2$ veces por semana & 53 \\
\hline Rara vez & 58 \\
\hline Nunca & 1 \\
\hline
\end{tabular}

Tabla 4. Agresiones más frecuentes

\begin{tabular}{|l|c|}
\hline \multicolumn{1}{|c|}{ AMBAS INSTITUCIONES } & TOTAL \\
\hline Manipulación - coacción & 4 \\
\hline Insultos y amenazas & 50 \\
\hline Maltrato físico & 129 \\
\hline Rechazo & 5 \\
\hline Otras formas & 10 \\
\hline
\end{tabular}


Tabla 5: Actitud ante el acoso.

\begin{tabular}{|l|c|}
\hline \multicolumn{1}{|c|}{ AMBAS ESCUELAS } & TOTAL \\
\hline Alejarse & 103 \\
\hline Responder con golpes & 17 \\
\hline Gritar & 20 \\
\hline Pedir ayuda & 83 \\
\hline
\end{tabular}

Tabla 6: Ayuda para superar el bulling.

\begin{tabular}{|l|c|}
\hline \multicolumn{1}{|c|}{ AMBAS INSTITUCIONES } & TOTAL \\
\hline Tu solo & 3 \\
\hline Amigos & 19 \\
\hline Docente del curso & 88 \\
\hline Otras personas & 10 \\
\hline Otro docente & 1 \\
\hline Directivos de la institución & 9 \\
\hline Padres & 64 \\
\hline
\end{tabular}

\section{Discusión de resultados}

En el análisis de los espacios donde son vulnerables a la situación del bullying el $43 \%$ considera que son más propenso a esta situación en el aula de clase, donde los apodos, mofas, son parte del proceso académico de acuerdo las situaciones.

Las agresiones son parte del diario vivir de los niños de la escuela Teodoro Wolf más que de la institución 24 de Julio, siendo más frecuente el maltrato verbal y psicologico en relacion a la discriminacion, separacion de grupo, exlusión de juegos.

Las victimas sufren más de maltrato fisico en un 65\% (empujones, manotones, aruños, estrujones, golpes). La explicacion se debe a que este grupo es más debil e inseguro por lo cual es presa facil del agresor. El porcentaje restante (25\%) recibe amenazas de ambito psicológico (insultos, apodos, ridulización por su forma de vestir, burlas de la apriencia fisica o forma de ser).

Las forma más usada para controlar el bullyng es alejandose en un $46 \%$, es decir, desarrollan una conducta evitativa, evaden el momento y no solucionan el problema, el $37 \%$ pide ayuda al docente, padres y el $8 \%$, la minoría, responde a la situación o confronta el problema.

Los estudiantes responden que el docente es el único que puede resolver esta situación en un $45 \%$, el $33 \%$ expresa que los padres, y el $10 \%$ refiere que sus amigos. Los demmas niños y niñas que observan estos hechos son sólo observadores pasivos, no se involucran por miedo pues temen convertirse en blanco de este problema.

\section{Conclusiones}

El bullying es un fenómeno no individual sino más bien grupal muchos niños este riesgo es demostrado al sentir temor, ansiedad o por ser intimidados constantemente se alejan o se enferma y evaden ir a la escuela contextualizada por la segregación y marginación en la convivencia diaria. Los docentes, a veces no saben cómo actuar frente a esta situación evadiendo el problema y consideran en acoso verbal o psicológico como algo normal.

El mayor rango de edad del bullying en la escuela es de 7 a 8 años y la violencia física de cualquier forma a veces pasa desapercibidos incluso hasta por los padres.

Se validad la necesidad de reconsiderar la elaboración de un programa donde las estrategias estén dirigidas a la prevención e intervención y pueda involucrarse a toda la comunidad educativa. Dentro de estos proyectos y de acuerdo a los resultados obtenidos se deben aplicar actividades recreativas representadas en teatro, juegos, ejercicios en que su base principal sean los valores personificados en situaciones diarias para tratar de minimizar el impacto de la escasa convivencia estudiantil y fortalecer la integración social.

Disponer de estas acciones para mejorar la convivencia estudiantil y tener identificadas las zonas más vulnerables de posible bullying, ubicando señaléticas y plan de difusión e información constante de prevención en las dos instituciones beneficiarias a través de las capacitaciones.

Promover una buena práctica de convivencia y el problema sea comprendido y dimensionado.

\section{Referencias}

1. Olweus, D. (1973). Personality and aggression. I J.K. Cole, \& D.D. Jensen (Eds.), Nebraska Symposium on Motivation 1972. Lincoln: University of Nebraska Press.

2. América Latina: violencia entre estudiantes y desempeño escolar Marcela Román y F. Javier Murillo, revista CEPAL 104. 2011. 
3. Bausela Herreras, E. Estrategias para prevenir el bullying en las aulas. Revista de Intervención Psicosocial, 2008. Vol. $17 \mathrm{~N} .^{\circ} 3$ Págs. $\quad 369-370 . \quad$ ISSN: 1132-055. Recuperado el 30 de septiembre de 201, de http://www.redalyc.org/pdf/1798/179814023 011.pdf.

4. CEMPROC. Centro de Mediación, Paz y Resolución de Conflictos del Ecuador. Recuperado el 17 de 12 de 2013, de http://www.cemproc.org/ecuador/

5. Díaz Aguado, M. Porque se produce la violencia escolar y como prevenirla. Revista Iberoamericana de Educación. N³7. 2005. OIE. Madrid. España, 17-47.

6. GAIRÍN, Joaquín. El «BULLYING» Escolar. Consideraciones. Revista Siglo XXI, 2013. Pp 17 a 38.
7. Herrera, B. Estrategia para Prevenir el Bullying en las Aulas: Intervención Psicológica. Madrid. 2008.

8. Ministerio de Educción acuerdo 3412- pdf. www.educacion.gob.ec:

https://educacion.gob.ec/wp-

content/uploads/downloads/2017/05/2-

Guia-Acoso-Escolar-tutores.pdf

9. Olweus, D. Conductas de acoso y amenazas entre escolares, Madrid, Ediciones Morata. 1998.

10.Semfyc. Tratado de Medicina de Familia y Comunitaria ( $1^{\underline{a}}$ edición ed.). (V. Casado, S. Calero, F. Cordón, E. De Serdio, M. Ezquerra, M. Gálvez, et al., Eds.) Barcelona: Semfyc ediciones. 2007 